\title{
Study of biofilm formation in Salmonella species isolated from food
}

\author{
Mohammad Mehdi Soltan Dallal ${ }^{1,2} \odot$, Mohammad Khalifeh-Gholi ${ }^{3,4} \odot$, Hojjat Rahmani ${ }^{\circ}$, Sara \\ Sharifi-yazdi ${ }^{6} \odot$, Shabnam Haghighat Khajavi ${ }^{7} \odot$, Mohammad Kazem Sharifi Yazdi ${ }^{8} \odot$
}

${ }^{1}$ Department of Pathobiology, Division of Microbiology, School of Public Health, Tehran University of Medical Sciences, Tehran, Iran ${ }^{2}$ Food Microbiology Research Center, Tehran University of Medical Sciences, Tehran, Iran

${ }^{3}$ Department of Microbiology and Immunology, Cellular and Molecular, Faculty of Medicine, Qom University of Medical Sciences, Qom, Iran

${ }^{4}$ Molecular Research Center, Qom University of Medical Sciences, Qom, Iran

5Department of Management Sciences and Health Economics, School of Public Health, Tehran University of Medical Sciences, Tehran, Iran

${ }^{6}$ School of Medicine, Tehran University of Medical Sciences, Tehran, Iran

${ }^{7}$ Department of Food Sciences and Technology, Science and Research Branch, Islamic Azad University, Tehran, Iran

${ }^{8}$ Department of Medical Laboratory Sciences, Zoonosis Research Center, Tehran University of Medical Sciences, Tehran, Iran

DOI: $10.18621 /$ eurj.434298

\section{ABSTRACT}

Objectives: Biofilms are defined as communities of organisms attached to a surface and producing an extracellular matrix, in which the bacteria are imbedded. Infections with Salmonella species represent a major health problem and a significant burden on food industry. Biofilm formation is one of the causes of pathogenicity of Salmonella species, especially in the food industry, which allows bacteria to bind to different levels. Many outbreaks have been associated with biofilms, because they quickly resist anti-microbial and cleansing agents. The aim of this research was to study the capability of biofilm formation by Salmonella species isolated from food.

Methods: A total of 8 Salmonella species were isolated from 400 samples of red meat, chicken, eggs, and vegetables. Identification was carried out by conventional biochemical tests and serotyping. The capability of biofilm production was measured by titration in Crystal Violet microplate.

Results: In the phenotypic study of Salmonella isolates with turbidity method at $550 \mathrm{~nm}$ without acetic acid, only $2(25 \%)$ of isolates were able to produce biofilm. both of isolates belonged to the group D of Salmonella. Conclusions: The capability of the isolates to form biofilm reveals the potential ability to resist antimicrobial chemotherapy, therefore higher levels of hygiene in production, packaging, and supply are necessary.

Keywords: Salmonella, biofilms, foodborne disease

Received: June 17, 2018; Accepted: March 2, 2019; Published Online: July 21, 2019

$\mathrm{T}$ he incidence of non-typhoidal salmonellosis in the United States is reported to be 1.4 million per year, with over $95 \%$ of these cases being foodborne diseases and $30 \%$ of these food infections results in death. Various studies have shown the high capability of Salmonella species to bind and form biofilm on different surfaces $[1,2]$. A biofilm is any group of microorganisms in which cells stick to each other and

Address for correspondence: Mohammad Kazem Sharifi Yazdi, MD., Tehran University of Medical Sciences, Department of Medical Laboratory Sciences, Zoonosis Research Center, Tehran, Iran, E-mail: mksharifiyazdi@gmail.com, Tel: 98218 8983919, Fax: 98218 8983919 
often also to a surface. These adherent cells become embedded within a slimy extracellular matrix that is composed of extracellular polymeric substances [3]. The formation of biofilms reduces the susceptibility to antimicrobial treatment which will ultimately lead to high treatment costs for patients [4]. Food contamination in the production line through unsanitary surfaces is one of the most common problems in food processing plants. Improperly cleaned and residue levels are a good environment for binding and growth, of pathogenic bacteria and, consequently, biofilms formation. The passage of the processed product from contaminated surfaces causes its microbial contamination $[5,6]$. The growth of bacteria in the biofilm on the surfaces makes it easier them to transport and difficult to eliminate them. Because biofilm cells exhibit greater resistance to biosolids and disinfectants compared with free cells $[7,8]$. The growth of biofilms on food processing equipment causes microbial contamination in the process product, thus reducing the shelf life of the product and increasing the prevalence of food-borne diseases, in particular, those related to Listeria monocytogenes and Salmonella species. These biofilm contain pathogenic microorganisms $[9,10]$. Since there was little information about the formation of biofilm from Salmonella species isolated from food in Iran the purpose of this study was to investigate thecapability of biofilm formation by Salmonella species isolated from food.

\section{METHODS}

A total of 8 Salmonella species were isolated from 400 samples of red meat, chicken, eggs, and vegetables. Identification was carried out by

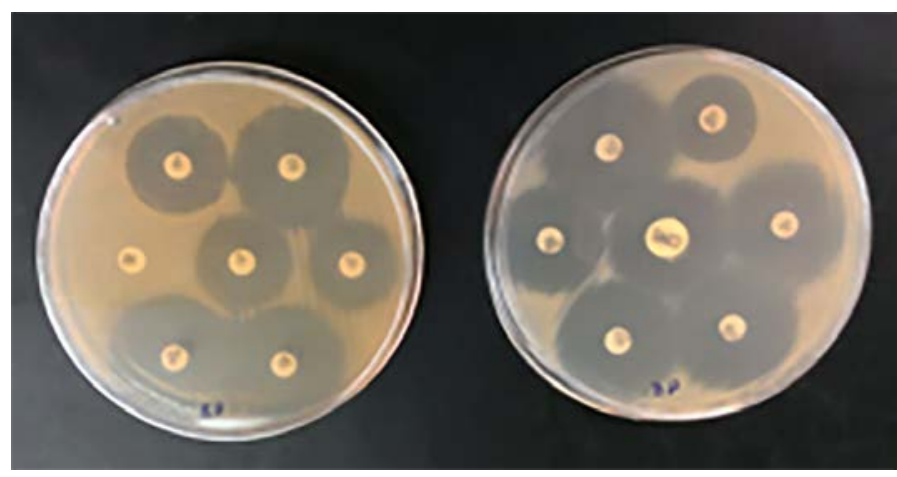

Figure 1. Antibiotic sensivity test. conventional biochemical tests and serotyping. Antibiotic sensitivity tests were carried out on the identified Salmonella species by using the KirbyBauer (Figure 1). Twelve antibiotic discs, namely amoxicillin, nalidixic acid, chloramphenicol, imipenem, tetracycline, ciprofloxacin, ceftriaxone, meropenem, streptomycin, cefepime, cefuroxim and cotrimoxazole. Results were analyzed according to Clinical and Laboratory Standards Institute (CLSI) [11].

\section{Biofilm Production}

The capability of biofilm production was measured by titration in Crystal Violet microplate according to the instructions used by Peeters et al. [12]. Samples was cultured in tryptic soy broth (TSB) and incubated at $37^{\circ} \mathrm{C}$ for 24 hours. After dilution in fresh TSB, $150 \mathrm{ml}$ of cell suspension was poured into a 96 well flat-bottom polystyrene microplate and incubated at $37^{\circ} \mathrm{C}$ for 24 hours. The plate was washed three times with $200 \mu 1$ of posphate-buffered saline (PBS) and air-dried. For fixation of biofilms, $100 \mu 1$ of $99 \%$ methanol was used, after 15 minutes, alcohol was removed and plates were dried in air. $100 \mu 1$ of $2 \%$ crystal violet was added to all of the wells and after 20 minutes the plates were washed with water to remove the color residues. The bonded colors were then released by adding $150 \mu \mathrm{L}$ of $33 \%$ acetic acid. The light absorption (OD) of each well was measured at $570 \mathrm{~nm}$ using the ELISA reader. All measurements were repeated 4 times .This was repeated in three separate experiments. E. coli Top 10 and E.coli EAEC 042 strains were used as a negative and positive control respectively.

\section{RESULTS}

Of the eight isolated Salmonella, two isolates had the capability to produce biofilms, both of which belong to group D (Figure 2). The Salmonella isolates showed, the highest resistance $6(75 \%)$ to nalidixic acid, $3(37.5 \%)$ were intermediate to ciprofloxacin and cefuroxime amoxicillin. All isolates $8(100 \%)$ were sensitive to chloramphenicol, imipenem, meropenem, ceftriaxone, cefepime, streptomycin, and cefotaxime. Serogroup D Salmonella has the highest resistance to nalidixic acid (75\%). Serogroup A was susceptible to 


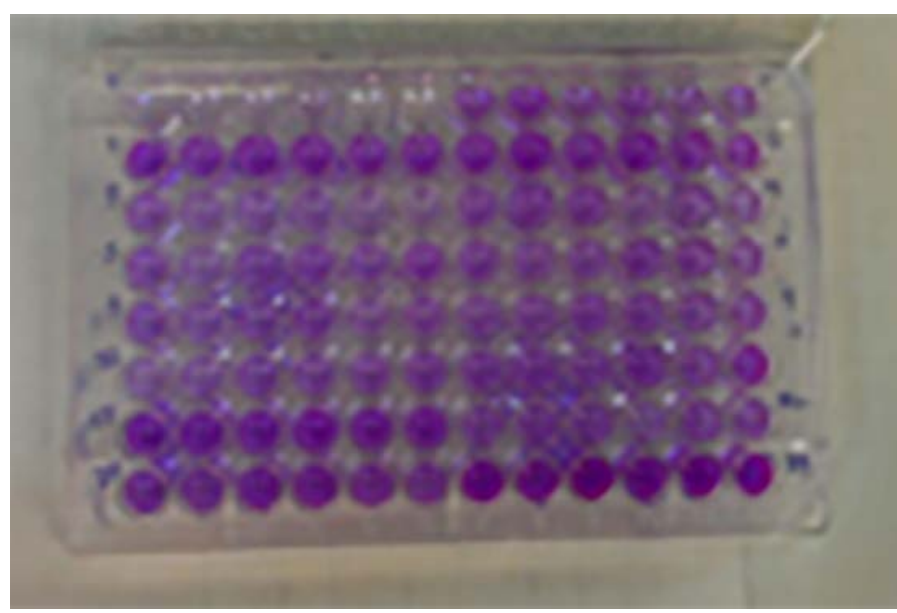

Figure 2. Biofilm production.

cefuroxime and nalidixic acid and intermediate to the rest of antibiotic. Salmonella serogroup B was resistant to nalidixic acid, tetracycline, cotrimoxazole, and amoxicillin and sensitive to the rest of the antibiotic. Salmonella serogroup C was resistant to nalidixic acid and tetracycline, intermediate to ciprofloxacin and sensitive to the rest of antibiotics. All non-typeable Salmonella showed 100\% sensitivity to the entire tested antibiotic (Table 1).

\section{DISCUSSION}

Salmonella is an important foodborne pathogen and its prevalence in fresh food poses a threat to human. The increase in demand and consumption of raw vegetables has resulted in a rise in food-borne related illnesses and outbreaks. The biofilm formation is a mechanism of Salmonella to adapt to different environments. They have been of considerable interest in food hygiene since biofilms may contain spoilage and pathogenic bacteria which increases postprocessing contamination and risk to public health. In addition, biofilm cells are more resistant to cleaning and disinfection processes in the food industry. A number of studies have shown that Salmonella spp. are capable of adhering and forming biofilms ondiverse surfaces including metal, glass and rubber surfaces [13-15]. The assessment of biofilm formation by Salmonella on microtitre plateshowed that all Salmonella isolates were able to form biofilms. Other research worker showed that the Salmonella were able to form biofilm on microtiter [16].

Pervious study also showed that Salmonella biofilms grown and established on stainless steel

Table 1. Antibiotic susceptibility profile of serogroup A, B, C \& D and non-typeable Salmonella.

\begin{tabular}{|c|c|c|c|c|c|c|c|c|c|c|}
\hline \multirow[t]{2}{*}{ Antibiotics } & 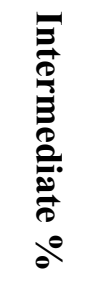 & 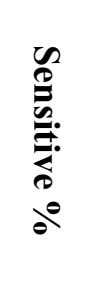 & 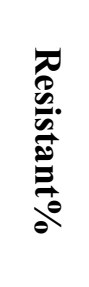 & 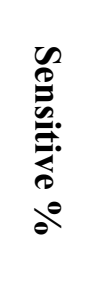 & 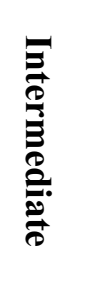 & 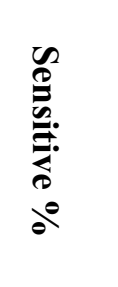 & 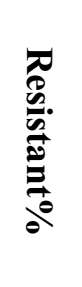 & 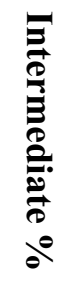 & 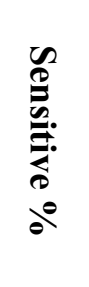 & \multirow{2}{*}{ 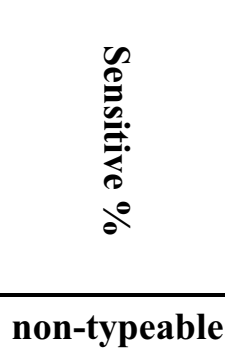 } \\
\hline & \multicolumn{2}{|c|}{ serogroup A } & \multicolumn{2}{|c|}{ serogroup B } & \multicolumn{2}{|c|}{ serogroup $C$} & \multicolumn{3}{|c|}{ serogroup D } & \\
\hline Amoxicillin & 100 & 0 & 100 & 0 & 0 & 0 & 100 & 100 & 0 & 100 \\
\hline Nalidixic acid & 0 & 100 & 100 & 0 & 0 & 100 & 0 & 0 & 25 & 100 \\
\hline Chloramphenicol & 100 & 0 & 0 & 100 & 0 & 0 & 100 & 0 & 100 & 100 \\
\hline Imipenem & 100 & 0 & 0 & 100 & 0 & 0 & 100 & 0 & 100 & 100 \\
\hline Tetracycline & 0 & 0 & 100 & 0 & 0 & 100 & 0 & 0 & 0 & 100 \\
\hline Ciprofloxacin & 100 & 0 & 0 & 100 & 100 & 0 & 0 & 100 & 0 & 100 \\
\hline Ceftriaxone & 100 & 0 & 0 & 100 & 0 & 0 & 100 & 0 & 100 & 100 \\
\hline Meropenem & 100 & 0 & 0 & 100 & 0 & 0 & 100 & 0 & 100 & 100 \\
\hline Streptomycin & 100 & 0 & 0 & 100 & 0 & 0 & 100 & 0 & 100 & 100 \\
\hline Cefepime & 100 & 0 & 0 & 100 & 0 & 0 & 100 & 0 & 100 & 100 \\
\hline Cefuroxime & 0 & 100 & 0 & 100 & 0 & 0 & 100 & 100 & 0 & 100 \\
\hline Cotrimoxazole & 0 & 0 & 100 & 0 & 0 & 0 & 100 & 0 & 0 & 100 \\
\hline
\end{tabular}


surfaces as well as meat thawing-loss broth (MTLB). This finding is a matter for concern, particularly for the poultry and meat processing industries using modern meat processing equipment. In these situations with mechanical and process automation, the surfaces are in repeated contact with raw meat, thusincreasing the opportunities for Salmonella transfer and attachment leading to biofilm formation [17].

The Salmonella isolates showed, the highest resistance $6(75 \%)$ to nalidixic acid, $3(37.5 \%)$ were intermediate to ciprofloxacin and cefuroxime amoxicillin. All isolates $8(100 \%)$ were sensitive to chloramphenicol, imipenem, meropenem, ceftriaxone, cefepime, streptomycin, and cefotaxime. Serogroup D Salmonella has the highest resistance to nalidixic acid (75\%). Serogroup A was susceptible to cefuroxime and nalidixic acid and intermediate to the rest of antibiotic. Several studies have documented high resistance of salmonella to the tetracyclines [18, 19], which is in agreement with the result obtained in this study. Salmonella serogroup B was resistant to nalidixic acid, tetracycline, cotrimoxazole, and amoxicillin and sensitive to the rest of the antibiotic. Salmonella serogroup C was resistant to nalidixic acid and tetracycline, intermediate to ciprofloxacin and sensitive to the rest of antibiotics. All non-typeable Salmonella showed $100 \%$ sensitivity to the entire tested antibiotic. A study carried in Canada showed the highest incidence of food-borne outbreaks, with the highest intake of vegetables and fresh fruits, with Salmonella with 50\% had the highest incidence of this disease, while Salmonella isolates from food were 2\% and vegetarians showed lower rates than chicken and meat [20]. A study a total of 48 strains of Salmonella enteritidis isolated from various sources in South America were investigated in terms of virulence factors including invasion, biofilm production, movement, presence of viral plasmid [21]. In this study, most strains were highly invasive and only three strains were low invasive. All the strains with low invasive did not produce biofilms, while $53 \%$ of high invasive produced biofilm [21]. In food industries, the binding of pathogenic bacteria and food corrosive to food contact levels in their production and packaging processes, and finally, the formation of microbial biofilms could be a potential source of contamination of food products and diseases and transmission of diseases. Biofilms on the surfaces of bacteria make it easier to transport and eliminate them. Because biofilm cells exhibit greater resistance to biosolids and disinfectants compared with free cells [7].

\section{CONCLUSION}

To consider the ability of producing biofilm by isolated salmonella from food samples and rising of salmonella gastroenteritis's especially group D, needing for more care and observance a higher level of health to preparation, producing, packing and supply of food seems.

\section{Conflict of interest}

The authors disclosed no conflict of interest during the preparation or publication of this manuscript.

\section{Acknowledgment}

This research was supported by Tehran University of Medical Sciences (TUMS) under contract number 32414. The authors thank the TUMS for their supportand Sara Pordel who help to implement this article.

\section{REFERENCES}

[1] Austin JW, Sanders G, Kay W, Collinson SK. Thin aggregative fimbriae enhance Salmonella enteritidis biofilm formation. FEMS Microbiol Lett 1998;162:295-301.

[2] Giaouris ED, Nychas GJ. The adherence of Salmonella enteritidis PT4 to stainless steel: The importance of the air-liquid interface and nutrient availability. J Food Microbiol 2006;23:74752.

[3] Duran N, Ozer B, Duran GG, Onlen Y, Demir C. Antibiotic resistance genes \& susceptibility patterns in staphylococci. Indian J Med Res 2012;135:389-96.

[4] Atshan SS, Nor Shamsudin M, Sekawi Z, Lung LT, Hamat RA, Karunanidhi A, et al. Prevalence of adhesion and regulation of biofilm-related genes in different clones of Staphylococcus aureus. J Biomed Biotechnol 2012;2012:976972.

[5] Van Houdt R, Michiels CW. Biofilm formation and the food industry, a focus on the bacterial outer surface. J Appl Microbiol 2010;109:1117-31.

[6] Palmer J, Flint S, Brooks J. Bacterial cell attachment, the beginning of a biofilm. J Ind Microbiol Biotechnol 2007;34:57788.

[7] Bryers JD. Biofilms II: Process analysis and application. New York: John Wiley and Sons, 2000.

[8] Bae YM, Baek SY, Lee SY. Resistance of pathogenic bacteria on the surface of stainless steel depending on attachment form 
and efficacy of chemical sanitizers. Int J Food Microbiol 2012;153:465-73.

[9] Wu Y, Park KC, Choi BG, Park JH, Yoon KS. The Antibiofilm effect of Ginkgo biloba extract against Salmonella and Listeria isolates from Poultry. Foodborne Pathog Dis 2016;13:229-38.

[10] Chen D, Zhao T, Doyle MP. Control of pathogens in biofilms on the surface of stainless steel by levulinic acid plus sodium dodecyl sulfate. Int J Food Microbiol 2015;207:1-7.

[11] Clinical and Laboratory Standards Institute. Performance standards for antimicrobial susceptibility testing; 25th informational supplement. M100-S25. Clinical and Laboratory Standards Institute, Wayne, PA, 2015;35(3):1-231.

[12] Peeters E, Nelis HJ, Coenye T. Comparison of multiple methods for quantification of microbial biofilms grown in microtiter plates. J Microbiol Methods 2008;72:157-65.

[13] Hood SK, Zottola EA. Adherence tostainless steel foodborne microorganisms during growth in model food systems. Int J Food Microbiol 1997;37:145-53.

[14] Joseph B, Otta SK, Karunasagar I, Karunasagar I. Biofilm formation by Salmonella spp. on food contact surfaces and their sensitivity to sanitizers. Int J Food Microbiol 2001;64:367-72.

[15] Sivapalasingam S, Friedman CR, Cohen L, Tauxe RV. Fresh produce: a growing cause of outbreaks of foodborne illness in the United States, 1973 through 1997. J Food Prot 2004;67:234253 .
[16] Igbinosa IH. Biofilm formation of Salmonella species isolated from fresh cabbage and spinach. J Appl Sci Environ Manag 2015;19:45-50.

[17] Wang H, Ding S, Wang G, Xu X, Zhou G. In situ characterization and analysis of Salmonella biofilm formation under meat processing environments using a combined microscopic and spectroscopic approach. Int J Food Microbiol 2013;167:293-302.

[18] Learn-Han L, Yoke-Kqueen C, Shiran MS, Sabrina S, Noor Zaleha AS, Sim JH, et al. Molecular characterization and antimicrobial resistance profiling of Salmonella enterica subsp. enterica isolated from 'Selom' (Oenanthe stolonifera). Int Food Res J 2009;16:191-202.

[19] Lertworapreecha M, Sutthimusik S, Tontikapong K. Antimicrobial resistance in Salmonella enterica isolated from pork, chicken, and vegetables in southern Thailand. Jundishapur J Microbiol 2013;6:36-41.

[20] Kozak GK, MacDonald D, Landry L, Farber JM. Foodborne outbreaks in Canada linked to produce: 2001 through 2009. J Food Prot 2003;76:173-83.

[21] Shah DH, Zhou X, Addwebi T, Davis MA, Orfe L, Call DR, et al. Cell invasion of poultry-associated Samonella enterica serovar Enteritidis isolates is associated with pathogenicity, motility and proteins secreted by the type III secretion system. Microbiology 211;157(Pt 5):1428-35. 